\title{
Support Vector Classification of Acute Myocardial Ischemia with Reduced Sets of Body Surface Potential Map Electrodes
}

\author{
T Konttila ${ }^{1}$, M Stenroos ${ }^{1}$, H Väänänen ${ }^{1}$, H Hänninen ${ }^{2}$, M Lindholm ${ }^{1}$, I Tierala ${ }^{2}$, T Katila ${ }^{1}$ \\ ${ }^{1}$ Laboratory of Biomedical Engineering, Helsinki University of Technology, Finland \\ ${ }^{2}$ Division of Cardiology, Helsinki University Central Hospital, Finland
}

\begin{abstract}
Body Surface Potential Maps (BSPM) can be used to localise supply ischemia [1]. The complete lead set could be used for more exact classification than with any reduced set, but for clinical purposes reduced sets are useful. In this work, three best unipolar ECG leads are determined for each pairwise classification between artery groups and non-ischemic control subjects.

Most suitable ECG parameters are first selected using interpatient variance. Separating hyperplanes are then found using linear kernel support vector machine (SVM). Success in classification with the hyperplane is used as an indicator of goodness of an electrode set. The best classifying channels are then located with simulated annealing.

The results indicate that in addition to close proximity to the heart, an electrode on the posterior thorax is needed for differentiating between arteries. Most of the resulting leads are found in the approximate directions of heart axes.
\end{abstract}

\section{Introduction}

Methods for disease diagnosis and monitoring using small sets of electrocardiographic leads have been introduced $[2,3]$. These methods approach the selection of correct leads by analysing the location of largest change in a parameter or the statistically most significant channel. A parameter is for example an integral or amplitude of a given time or interval for each channel.

This work concentrates on finding a reduced set of electrodes that can be used to linearly classify myocardial ischemia using BSPMs measured during balloon angioplasty. For finding the best linear separator, a linear kernel support vector machine (SVM) is used with simulated annealing. This linear separator can then be used even without using the support vector machine as a black-box approach. Three best ECG leads are located for pairwise classification between each four conditions; non-ischemic, left anterior descending (LAD) artery, circumflex (LCx) and right coronary artery (RCA) ischemia. This results at maximum in 18 unipolar leads.

\section{Methods}

\subsection{Patient population}

Both the training and testing sets were produced from balloon angioplasty patients, represented by 8 LAD patients, 7 LCx patients and 7 RCA patients. In addition 8 non-ischemic subjects were selected as the control group. From each patient 41 beats were selected during angioplasty, and four subjects were randomly selected from each artery group to be the training group. This results in 164 training samples for each artery group. Non-ischemic and LAD groups have 164 testing samples while LCx and RCA groups have 123 testing samples.

The testing samples are used for detecting the best channel sets for linear classification. Because of the small number of patients, an independent test group is missing.

\subsection{Body surface potential mapping}

The Helsinki BSPM layout is presented in Fig. 1. It contains 120 chest leads and three limb leads. [4]

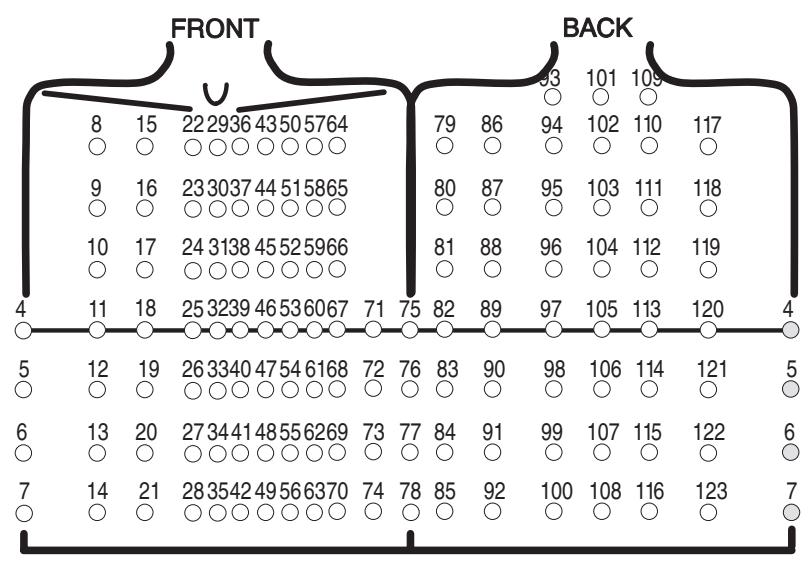

Figure 1. The Helsinki BSPM layout 


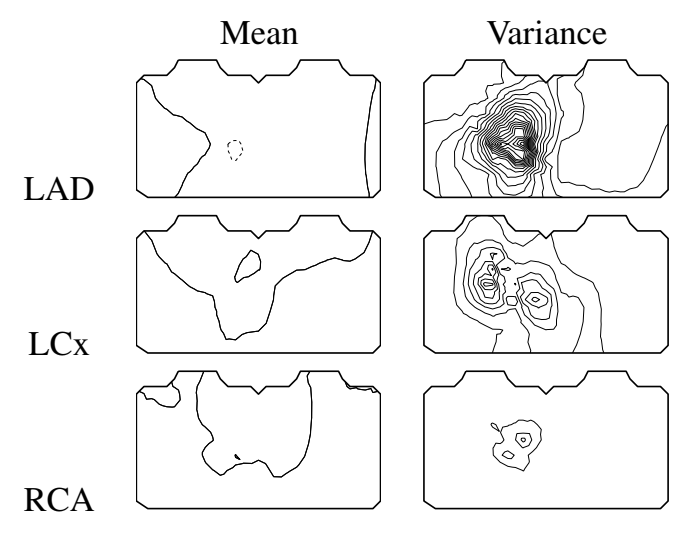

Figure 2. Mean and variance QRS integral maps (in scale)

\subsection{Patient to patient variance}

Suitability of a parameter was measured as the interpatient stability of the change in parameter during balloon angioplasty. The state before balloon was set as baseline map. On the whole, 34 parameters were tested. Out of this set 10 were depolarisation related and 24 were STsegment or repolarisation related. The example means and variances can be seen in Fig. 2 and 3. This indicated that the good parameters for differentiating purpose are, as expected, ST-segment and repolarisation amplitudes.

\subsection{Selected parameters}

Three ST-segment parameters were selected; J-point amplitude and amplitudes at $60 \mathrm{~ms}$ and $80 \mathrm{~ms}$ after J-point (ST60 and ST80). In addition, a repolarisation parameter, T-wave amplitude, was selected (Fig. 4).

\subsection{Support vector classification}

Support vector machine (SVM) is a powerful and universal learning machine [5]. In this case the separating efficiency has been limited to get easily interpretable results. Basic idea of SVM is to find a separating hyperplane between classes that maximizes the margin between classes. Normally the support vector machine uses nonlinear mapping to higher dimensional space to increase the probability of linear separation of patterns in accordance with Cover's theorem [6], which in this case has been limited by requiring linearity. Nonlinear mapping could effectively increase the Vapnik-Chervonenkis (VC) dimension to infinity, but still the generalising ability of SVM remains good. It is suspected that this is the result of the maximal margin between classes [5].

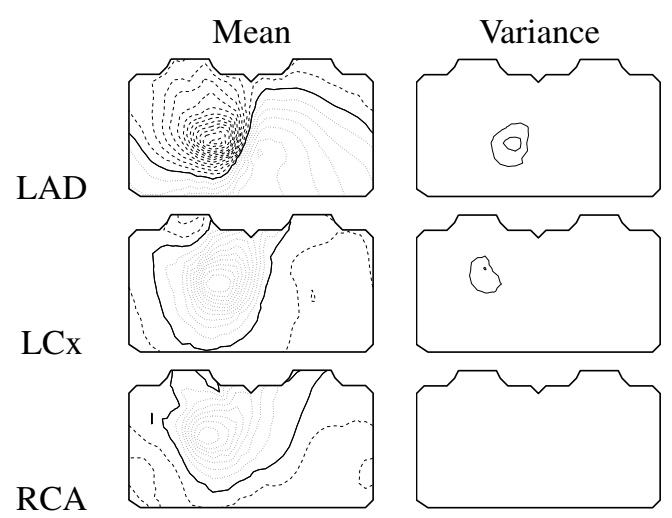

Figure 3. Mean and variance ST60 maps (in scale)

According to [5], the classification function is

$$
f(\mathbf{x})=\phi(\mathbf{x}) \cdot \mathbf{w}+b=\sum_{i=1}^{M} y_{i} \alpha_{i} \phi(\mathbf{x}) \cdot \phi\left(\mathbf{x}_{\mathbf{i}}\right)+b,
$$

where $\phi(\mathbf{x})$ is the nonlinear projection, which reduces to $\mathbf{x}$ in this case, $y_{i}$ is the class of training vector $i$ and $y_{i} \in$ $\{-1,1\}, \mathbf{x}$ is the unknown vector, and $\mathbf{x}_{\mathbf{i}}$ is the $i$ :th training vector. $\alpha_{i}$ is the weight parameter of training vector $\mathbf{x}_{\mathbf{i}}$ and $M$ is the number of training vectors. Note that $\alpha_{i} \geq 0$ and thus all of the training vectors do not have weight. Those vectors that have weight are called support vectors. In this case (1) reduces to

$$
f(\mathbf{x})=\sum_{i=1}^{M} y_{i} \alpha_{i} \mathbf{x} \cdot \mathbf{x}_{i}+b .
$$

A method for solution of $\alpha_{i}$ and $b$ can be found in [5] and an implementation for SVM in [7].

\subsection{Simulated annealing}

Simulated Annealing [8] is a commonly used optimisation method. The idea is to first melt the system in high temperature and then gradually lower the temperature until the system freezes. In this case a walker is set to three $(l=3)$ electrodes randomly, and temperature set to 0.1 . In each step $l$ randomly selected walkers can move randomly

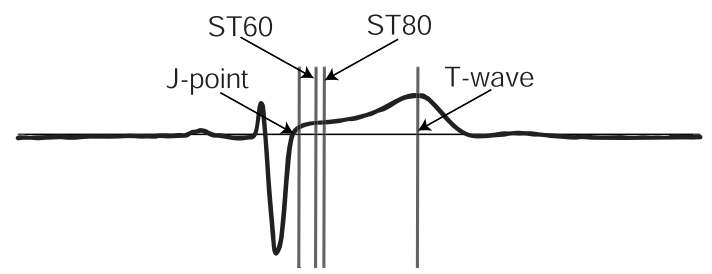

Figure 4. Selected parameters 
to their neighbouring electrodes (as in 4-neighbourhood). If the classification percentage is better with the new set of electrodes, it is automatically accepted. Otherwise, the step is accepted with probability

$$
p(\Delta c)=\exp \left\{-\frac{\Delta c}{T}\right\}
$$

where

$$
\Delta c=\frac{1}{N} \sum_{j=1}^{N} g\left(\mathbf{z}_{n-1}^{j}\right)-\frac{1}{N} \sum_{j=1}^{N} g\left(\mathbf{z}_{n}^{j}\right)
$$

and

$$
g(\mathbf{z})=\left\{\begin{array}{ll}
1 & \text { if } y_{j} f(\mathbf{z}) \geq 0 \\
0 & \text { if } y_{j} f(\mathbf{z})<0
\end{array} .\right.
$$

$\mathbf{z}_{n}^{j}$ is a vector containing the values of selected electrodes of testing patient $j$ at step $n$ and $N$ is the number of testing vectors. Note that $\Delta c \in(0,1]$. This step is repeated $10 l$ times and then the temperature is then lowered by $1 \%$, which slows the cooling enough for it to find a steady state. It is expected that the simulated annealing converges to a more convex solution.

\section{Results}

Tables 1-4 reflect the channels where simulated annealing converged. The simulated annealing was repeated three times to verify the results. Small variation was detected, especially with those instances where $100 \%$ accuracy was gained from multiple adjacent lead combinations. Most obviously this was the case with $\mathrm{LCx} / \mathrm{RCA}$ T-amplitude maps.

Table 1. J-point amplitude results

\begin{tabular}{lllll}
\hline \hline Arteries & Chn 1 & Chn 2 & Chn 3 & Test acc. \\
\hline LAD/CTRL & 40 & 52 & 83 & 0.926829 \\
LCx/CTRL & 47 & 62 & 37 & 0.933798 \\
RCA/CTRL & 47 & 96 & 71 & 0.864111 \\
\hline LAD/LCx & 13 & 60 & 99 & 0.940767 \\
LAD/RCA & 54 & 74 & 106 & 0.954704 \\
LCx/RCA & 45 & 63 & 61 & 0.869919 \\
\hline
\end{tabular}

Table 2. ST60 results

\begin{tabular}{lllll}
\hline \hline Arteries & Chn 1 & Chn 2 & Chn 3 & Test acc. \\
\hline LAD/CTRL & $\mathbf{5 1}$ & $\mathbf{4 6}$ & $\mathbf{9 5}$ & $\mathbf{0 . 9 7 8 6 5 9}$ \\
LCX/CTRL & 71 & 104 & 73 & 0.937282 \\
RCA/CTRL & 120 & 76 & 63 & 0.954704 \\
\hline LAD/LCx & 29 & 98 & 104 & 0.909408 \\
LAD/RCA & $\mathbf{4 3}$ & $\mathbf{5}$ & $\mathbf{3 8}$ & $\mathbf{0 . 9 7 9 0 9 4}$ \\
LCx/RCA & 18 & 85 & 76 & 0.821138 \\
\hline
\end{tabular}

\subsection{J-point amplitude}

The J-point amplitude (Table 1) analysis resulted in lower accuracy and stability in all arteries than any of the other parameters. Therefore it is not part of the final set of electrodes.

\subsection{ST60 amplitude}

The ST60 (Table 2) is one of the most common parameters for ischemia detection, and in this case it works best with LAD detection. In general, LAD is detected best in the proximity of the heart. With this patient set, ST60 is also most accurate in differentiating between LAD and RCA patients.

\subsection{ST80 amplitude}

The ST80 (Table 3) is a slightly modified version of the ST60, and works also in confirming the ST60 results. LAD vs. LCX are best detected with ST80.

\subsection{T-amplitude}

T-amplitude (Table 4) separates the LCx and RCA arteries. This could be the result of small amount of patients, but the simulated annealing quickly converges to 1 , and finds many different sets of electrodes for correct classification. Other good classification results are the separation of LCx and RCA from controls.

Table 3. ST80 results

\begin{tabular}{lllll}
\hline \hline Arteries & Chn 1 & Chn 2 & Chn 3 & Test acc. \\
\hline LAD/CTRL & 33 & 8 & 104 & 0.817073 \\
LCx/CTRL & 43 & 123 & 71 & 0.968641 \\
RCA/CTRL & 29 & 71 & 37 & 0.996516 \\
\hline LAD/LCx & $\mathbf{1 0 5}$ & $\mathbf{8 4}$ & $\mathbf{7 4}$ & $\mathbf{0 . 9 6 5 1 5 7}$ \\
LAD/RCA & 66 & 32 & 89 & 0.975610 \\
LCx/RCA & 84 & 30 & 43 & 0.947154 \\
\hline
\end{tabular}

Table 4. T-amplitude results

\begin{tabular}{lllll}
\hline \hline Arteries & Chn 1 & Chn 2 & Chn 3 & Test acc. \\
\hline LAD/CTRL & 105 & 28 & 7 & 0.969512 \\
LCx/CTRL & $\mathbf{6 7}$ & $\mathbf{3 5}$ & $\mathbf{4 7}$ & $\mathbf{0 . 9 9 6 5 1 6}$ \\
RCA/CTRL & $\mathbf{4 5}$ & $\mathbf{9 5}$ & $\mathbf{5 7}$ & $\mathbf{0 . 9 9 6 5 1 6}$ \\
\hline LAD/LCx & 112 & 12 & 87 & 0.909408 \\
LAD/RCA & 33 & 75 & 79 & 0.968641 \\
LCx/RCA & $\mathbf{2 7}$ & $\mathbf{5 4}$ & $\mathbf{1 2}$ & $\mathbf{1 . 0 0 0 0 0 0}$ \\
\hline
\end{tabular}



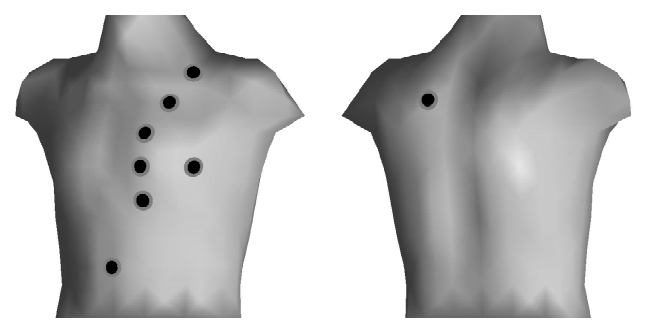

Figure 5. The layout for detection
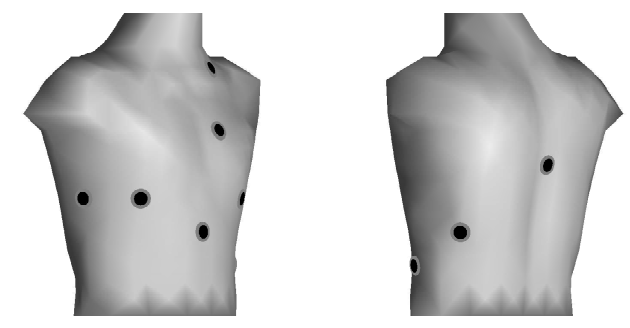

Figure 6. The layout for localisation

\subsection{The resulting leads}

From the results above, the complete set is as presented in Table 5. The lead set optimised for ischemia detection is presented in Fig. 5 and the set for ischemia localisation in Fig. 6. These results show that detection leads are concentrated in the precordial area with one lead on the anterior thorax. Localisation leads are distributed around the whole thorax.

\section{Discussion and conclusions}

The resulting leads for detection are positioned on the anterior thorax, while the localisation leads are distributed around the whole thorax. It can be argued that the leads needed for ischemia detection are the leads closest to the heart recording the strongest signal. Even the posterior detection lead is positioned close to the heart.

The locator leads on the other hand are located evenly around the whole thorax. It can be argued that the distal areas are more sensitive to the nature of change during ischemic episode. The changes are detected during any kind of ischemia in the proximity of the heart, but the change is similar in all cases.

This lead set is optimal for detection and localisation of ischemia with the given set of patients. On the other hand there are many unaccounted diseases and conditions for which this lead set may not be ideal.

The method introduced in this work was applied to the identification of the channels needed for accurate ischemia separation from non-ischemic patients and localising
Table 5. Resulting leads

\begin{tabular}{lllll}
\hline \hline Arteries & Parameter & Chn 1. & Chn 2. & Chn 3. \\
\hline LAD/CTRL & ST60 & 46 & 51 & 95 \\
LCx/CTRL & T & 35 & 47 & 67 \\
RCA/CTRL & T & 45 & 57 & 95 \\
\hline LAD/LCx & ST80 & 74 & 84 & 105 \\
LAD/RCA & ST60 & 5 & 38 & 43 \\
LCx/RCA & T & 12 & 27 & 54 \\
\hline
\end{tabular}

between the three main arteries. Any condition could be used with this method. The next phase would be to allow the channel to shift in given time steps in addition to the steps on torso surface. The method could also be used to detect channels that best separate between many classes simultaneously. These improvements combined with additional groups of patients would give valuable information of the most important recording locations.

\section{References}

[1] Horáček BM, Wagner GS. Electrocardiographic ST-segment changes during acute myocardial ischemia. Card Electrophysiol Rev 2002;6:196-203.

[2] Kornreich F. Identification of best electrocardiographic leads for diagnosing acute myocardial ischemia. J Electrocardiol 1998;31(Suppl):157-163.

[3] Horáček BM, Warren JW, Penney CJ, MacLeod RS, Title LM, Gardner MJ, Feldman CL. Optimal electrocardiographic leads for detecting acute myocardial ischemia. J Electrocardiol 2001;34(Suppl):97-111.

[4] Simelius K. Development of cardiographic mapping techniques for clinical use. Licentiate thesis, Department of Engineering Physics and Mathematics, Helsinki University of Tecnology, 1998.

[5] Cortes C, Vapnik V. Support-vector networks. Machine Learning 1995;20:273-297.

[6] Haykin S. Neural Networks: A Comprehensive Foundation. Upper Saddle River, NJ: Prentice Hall, 1999.

[7] Chang CC, Lin CJ. LIBSVM: a library for support vector machines, 2001. Software available at http://www.csie.ntu.edu.tw/ cjlin/libsvm.

[8] Kirkpatrick S, Gelatt Jr. CD, Vecchi MP. Optimization by simulated annealing. Science 1983;220:671-680.

Address for correspondence:

Teijo Konttila

Helsinki University of Technology

P.O.B 2200, 02015 HUT, Finland

tel.: +358-(0)9-451-2915

teijo.konttila@tkk.fi 